\title{
Anemia ferropriva e o uso do sulfato ferroso: facilidades e dificuldades na prevenção
}

\author{
Iron-deficiency anemia and use of ferrous sulfate: prevention facilitators and difficulties \\ Anemia ferropénica y el uso del sulfato ferroso: facilidades y dificultades en la prevención
}

\author{
Nayara Cristina Pereira Henrique', Paula Carolina Bejo Wolkers", Maria Cândida de Carvalho Furtado'", \\ Aurea Tamami Minagawa Toriyama", Débora Falleiros de Mellov
}

\begin{abstract}
RESUMO
Objetivo: apreender os saberes de mães/cuidadores com relação à anemia ferropriva e o uso preventivo do sulfato ferroso, com ênfase nos elementos que facilitam e dificultam a prevenção da enfermidade. Método: estudo exploratório qualitativo com análise temática indutiva dos dados, realizado por meio de entrevistas com 12 mães/cuidadores de crianças entre seis meses e 11 meses e 29 dias de idade, em acompanhamento em unidade de saúde da família de um município brasileiro. Resultados: a alimentação saudável foi apontada como elemento facilitador na prevenção da anemia ferropriva. Fragilidades nos conhecimentos sobre a enfermidade e o sulfato ferroso como estratégia preventiva, falhas na prescrição do medicamento e uso cotidiano, e poucas orientações foram os elementos que dificultam a prevenção da doença. Conclusão: práticas educativas em saúde na prevenção da anemia ferropriva são essenciais, sinalizando informações sobre a enfermidade e o monitoramento da suplementação profilática, fortalecendo o cuidado da saúde da criança.

Descritores: Criança; anemia ferropriva; prevenção; sulfato ferroso.
\end{abstract}

\section{ABSTRACT}

Objective: to learn about mothers' and caregivers' knowledge in relation to iron-deficiency anemia and preventive use of ferrous sulfate, with emphasis on factors that facilitate and hinder prevention of the disease. Method: in this qualitative exploratory study, stimulated thematic data analysis was used after interviews of 12 mothers and caregivers of children from six to 11 months and 29 days of age attending a family health unit in a Brazilian municipality. Results: healthy eating was indicated as a facilitator of iron-deficiency anemia prevention. Weaknesses in knowledge about the disease and about use of ferrous sulfate as a preventive strategy, flaws in medication prescription and daily use, and a scarcity of guidelines were the factors hindering prevention of the disease. Conclusion: health education activities in iron-deficiency anemia prevention, that signal information about the disease and monitoring of prophylactic supplementation, are essential to strengthening children's health care.

Keywords: Child; iron deficiency anemia; prevention; ferrous sulphate.

\section{RESUMEN}

Objetivo: entender los saberes de madres / cuidadores con relación a la anemia ferropénica y el uso preventivo del sulfato ferroso, con énfasis en los elementos que facilitan y dificultan la prevención de la enfermedad. Método: estudio exploratorio cualitativo con análisis temático inductivo de los datos, realizado por medio de entrevistas junto a 12 madres / cuidadores de niños entre seis y 11 meses y 29 días de edad, en seguimiento en unidad de salud de la familia de un municipio brasileño. Resultados: se apuntó la alimentación saludable como elemento facilitador en la prevención de la anemia ferropénica. Debilidades en los conocimientos sobre la enfermedad y el sulfato ferroso como estrategia preventiva, fallas en la prescripción del medicamento y uso cotidiano, y pocas orientaciones fueron los elementos que dificultan la prevención de la enfermedad. Conclusión: prácticas educativas en salud en la prevención de la anemia ferropénica son esenciales, señalando informaciones sobre la enfermedad y el monitoreo de la suplementación profiláctica, fortaleciendo el cuidado de la salud del niño.

Descriptores: Niño; anemia ferropriva; prevención; sulfato ferroso.

\section{INTRODUÇÃO}

A anemia causada por deficiência de ferro, denominada anemia ferropriva ( $\mathrm{AF})$, é a mais comum e considerada importante problema de saúde pública, por apresentar elevadas prevalências, bem como con- sequências adversas ${ }^{1,2}$, como comprometimento do sistema imunológico, aumento das taxas de mortalidade infantil, redução da função cognitiva, do crescimento e do desenvolvimento neuropsicomotor da criança ${ }^{3}$.

'Enfermeira. Mestre em Ciências pelo Programa de Pós-Graduação Enfermagem em Saúde Pública da Escola de Enfermagem de Ribeirão Preto- USP, Ribeirão Preto, São Paulo, Brasil. E-mail: nayara.henrique@usp.br

"Enfermeira. Doutora em Ciências. Enfermeira no Ambulatório de Pediatria e Neonatologia do Hospital das Clínicas da Universidade Federal de Uberlância MG. Brasil. E-mail: paulawolkers@yahoo.com.br.

"'Enfermeira. Professora do Departamento de Enfermagem Materno Infantil e Saúde Pública da Escola de Enfermagem de Ribeirão Preto-USP, Ribeirão Preto, São Paulo, Brasil. E-mail: mcandida@eerp.usp.br.

IVEnfermeira.Professor Doutor, Escola de Enfermagem, Universidade de São Paulo, São Paulo, SP, Brasil. E-mail: aureatmt@usp.br.

${ }^{\vee}$ Enfermeira. Professora Associada do Departamento de Enfermagem Materno Infantil e Saúde Pública da Escola de Enfermagem de Ribeirão Preto-USP, Ribeirão

Preto, São Paulo, Brasil. E-mail: defmello@eerp.usp.br. 
Em pesquisa brasileira de demografia e saúde da criança ${ }^{4}$, verificou-se maior prevalência da doença em $21,4 \%$ das crianças com idade inferior a 24 meses. Na infância, as principais causas da AF são as elevadas necessidades de ferro, somadas ao consumo insuficiente do mineral ${ }^{3}$.

A associação entre suplementação de ferro, fortificação de alimentos e educação alimentar vem sendo apontada como a melhor forma de prevenção da doença ${ }^{5}$. O Ministério da Saúde, visando à diminuição dos casos de anemia, desenvolve ações com destaque para o Programa Nacional de Suplementação de Ferro (PNSF), cujo objetivo principal é a redução dos casos e a prevenção da $A F$, essencialmente na faixa etária até 18 meses, como também em mulheres grávidas, por meio do uso do sulfato ferroso ${ }^{3,6}$, sendo este apontado como econômico, eficaz, tradicional e rápido para o controle da deficiência de ferro ${ }^{7}$.

Na execução de programas de atenção à saúde da criança, a baixa adesão a medidas preventivas da $A F$, especialmente ao uso do sulfato ferroso, é preocupante. Investigações ${ }^{3,4}$ evidenciaram que cerca de um terço das mães entrevistadas administraram medicamentos com ferro para as crianças, abaixo do proposto nas recomendações programáticas. Ao analisarem os conhecimentos de mães sobre a AF e suas formas de prevenção, resultado $s^{8,9}$ evidenciaram que $20,5 \%$ delas não souberam relatar nenhuma atividade de prevenção da anemia e algumas mostraram pouco conhecimento sobre a finalidade da suplementação de ferro com sulfato ferroso. Acresce-se, ainda, como fatores para a baixa adesão ao uso do sulfato ferroso, os efeitos colaterais apresentados pelas crianças e o esquecimento em administrar o medicamento ${ }^{8,10}$.

Isto posto, cabe apontar que a Estratégia Saúde da Família (ESF) tem como uma de suas ações prioritárias, na atenção à saúde da criança, o acompanhamento do crescimento e desenvolvimento infantil. Considerando que, se não tratada, a AF pode trazer complicações no crescimento e desenvolvimento infantil ${ }^{3,11}$, é importante que as equipes de ESF monitorem a adesão ao uso do sulfato ferroso e orientem as famílias sobre a AF e suas formas de prevenção. Admitindo que a AF se configura como um problema de saúde pública na infância, o objetivo deste estudo é apreender os saberes de mães/cuidadores com relação à $A F$ e sua prevenção, com destaque para o uso preventivo do sulfato ferroso e os elementos que facilitam ou dificultam a prevenção da enfermidade.

\section{MÉTODO}

Trata-se de um estudo exploratório com análise qualitativa dos dados, desenvolvido em um município do interior do estado de São Paulo.

O estudo foi realizado entre os meses de outubro e dezembro de 2015, em uma Unidade Saúde da Família (USF), escolhida pelo número expressivo de crianças menores de um ano de idade em seguimento na unidade, bem como por ser uma unidade com ESF desde 2001.
Participaram mães/cuidadores que atenderam aos seguintes critérios de inclusão: mães/cuidadores maiores de 18 anos que cuidavam das crianças em sua rotina diária, com crianças na faixa etária entre 6 e 11 meses e 29 dias no período da coleta de dados, em seguimento na ESF. A escolha por esta faixa etária escolhida justifica-se a partir das recomendações para o início da suplementação profilática de ferro pela Sociedade Brasileira de Pediatria (SBP) e pelo Ministério da Saúde ${ }^{3,12}$. Assim, a partir dos 12 meses de idade tem-se a recomendação de que as crianças estejam com a alimentação rotineira da família ${ }^{13,14}$.Os critérios de exclusão foram: crianças que interromperam o seguimento na ESF, mudança da área de abrangência da unidade ESF, crianças com tratamento para diferentes tipos de anemia diagnosticada ou outras patologias e crianças prematuras.

Para a coleta de dados, utilizou-se entrevista orientada por um roteiro semiestruturado com os principais cuidadores das crianças, com perguntas sobre a saúde da criança, a AF e suas formas de prevenção. Após um levantamento realizado na USF, identificou-se 16 mães/ cuidadores elegíveis; após a aplicação dos critérios, o estudo foi composto por 12 mães/cuidadores. As participantes foram identificadas com a letra $E$, seguida da ordem das entrevistas: E1, E2...E12. Os relatos foram audiogravados e realizados individualmente durante visitas domiciliares ou após consulta de puericultura.

As entrevistas foram conduzidas pela pesquisadora primeira autora, com uma média de duração de 30 minutos. $O$ início da entrevista foi guiado pela questão “Como está a saúde do(a) seu(sua) filho(a)?" e, posteriormente, com as perguntas: "O que significa anemia ferropriva para você?", "Como devem ser cuidadas as crianças para prevenir a anemia?", "Você conhece o medicamento sulfato ferroso?". A partir do encadeamento da entrevista, perguntas adicionais relacionadas à alimentação da criança, uso ou não do sulfato ferroso e experiência no uso do medicamento também foram realizadas. Das 12 entrevistas, três foram realizadas após a consulta de puericultura na unidade de saúde e nove por meio de visitas domiciliares.

A análise dos dados foi baseada na análise de conteúdo do tipo temática indutiva, em que os temas identificados são extraídos dos próprios dados ${ }^{15}$.

A dissertação de mestrado da qual se originou o presente estudo recebeu aprovação do Comitê de Ética em Pesquisa da Escola de Enfermagem de Ribeirão Preto-USP, conforme Ofício 131/2016, CAAE no 51885115.8.0000.5393. Utilizou-se o Termo de Consentimento Livre e Esclarecido, conforme a Resolução 466/12 do Conselho Nacional de Saúde.

\section{RESULTADOS}

Das doze entrevistas realizadas, onze foram com mães e uma com a avó materna, as quais se consideravam cuidadoras principais da criança e que as levavam para acompanhamento de saúde na referida unidade. 
Houve maior ocorrência da faixa etária entre 20 a 34 anos no momento do parto, da ocupação de dona de casa e, somente três entrevistadas possuíam sete ou mais anos de estudo. Com relação às crianças, oito eram do sexo masculino, nascidos de parto normal; uma das crianças nasceu com peso abaixo de $2500 \mathrm{~g}$, três tiveram aleitamento materno exclusivo até os seis meses, e para uma criança houve introdução alimentar tardiamente, com sete meses de idade.

Na análise dos dados, emergiram aspectos que traduzem os principais elementos do foco da investigação e foram agrupados nas seguintes unidades temáticas: "A anemia ferropriva: saberes sobre a enfermidade"; e "A prevenção da anemia e o uso do sulfato ferroso".

\section{A anemia ferropriva: saberes sobre a enfermidade}

Em seus relatos, as cuidadoras expressaram conhecimento sobre a AF e relacionaram a doença com problemas sanguíneos; porém, não especificam o problema e nota-se pouco aprofundamento com relação ao tema.

Eu sei que é falta de um ferro, mas não sei te explicar ao certo. (E1)

É a falta de ferro no corpo. (E5)

Acho que é [...] não é um problema com sangue? É a falta de sangue ou algo assim? (E4)

O que eu sei é que a gente precisa de mais ferro. É uma coisa no sangue, né? (E11)

Surge também a relação entre anemia e alimentação e alguns sintomas.

Para mim significa a criança não comer algumas coisas que contem ferro. Ai, por isso, surge a anemia. (E2)

Eu acho que anemia é uma má alimentação. Quando a pessoa se alimenta mal, a pessoa fica com anemia. (E7)

É quando a criança não come. A criança vai emagrecendo. Eu acho que é isso. (E9)

É quando você não come verdura, essas coisas, legumes. (E10)

A minha amiga, a filha dela já teve. E o olhinho dela aqui ficou branco, sabe? (E12)

Apesar de expressarem o conhecimento sobre a AF doença, é importante destacar falas coma associação entre a doença e o aparecimento de neoplasias.

A anemia é quando o sangue fica fraco, na minha opinião, e vira câncer de sangue, né? (E6)

Anemia é uma coisa muito séria, causa câncer se não prevenir, se não cuidar. Então, é perigoso se dá anemia. (E3)

Preocupação com AF devido a experiências prévias com a doença, foi vivenciada por uma das entrevistadas e relacionada, por outra, a algum parente/conhecido que já foi acometido pela doença.

Eu tenho muito medo de anemia, porque eu tive antes, na minha gravidez. (E2)

A minha mãe já teve anemia. Então, é uma coisa, pra mim, muito preocupante. Ela passou muito mal, desmaiava. Nossa, sinceramente, fiquei muito preocupada. (E12)

\section{A prevenção da anemia e o uso do sulfato ferroso}

Seis participantes relataram não haver prescrição de sulfato ferroso para a criança; as demais faziam uso no momento da entrevista ou já haviam utilizado o medicamento. Dessas, houve prescrição para uma criança aos sete meses de idade; para as demais, a prescrição seguiu as recomendações da Sociedade Brasileira de Pediatria e do Ministério da Saúde ${ }^{3,12}$.

No âmbito da prevenção da $A F$, algumas cuidadoras indicam, como estratégia, a alimentação saudável e um regular acompanhamento da saúde.

A alimentação é importante para prevenir, para ter proteínas, vitaminas, que estão presentes nas frutas e nos legumes. Eu acho que o ferro também é, vem das frutas, dos legumes e das carnes. (E1)

A alimentação e um acompanhamento médico. Acho que só uma boa alimentação já ajuda bastante. (E8)

Os alimentos que combatam a doença são muito importantes, e o cuidado com a alimentação. (E11)

Ainda com relação à prevenção da doença, houve o reconhecimento da importância do uso de um "medicamento", e em outros momentos dúvidas acerca deste medicamento. Denota-se, em algumas falas, que cuidadoras conhecem o medicamento pelo nome (sulfato ferroso). Além disso, percebem-se crenças e dúvidas de que uma alimentação saudável pode substituir a necessidade de utilização do sulfato ferroso.

Tem que dar o sulfato ferroso, né? E também tem que dar beterraba, bastante verdura. (E6)

Se você está prevenindo, dando a ele o remédio [Sulfato ferroso] antes do problema, isso é o melhor. É isso que é a melhor coisa a ser feita. A razão pela qual eu apoiei o sulfato ferroso é porque ele não está comendo bem. Se alguém come bem e descansa bem, você come no horário certo, você descansa na hora certa, sim está ok, não há necessidade de sulfato ferroso. Mas, se alguém não está comendo, então tem a necessidade de prevenir. (E4)

Algum remédio, se tivesse algum remédio pra dar, pra evitar, entendeu? Seria muito importante para prevenir. (E7)

Algumas entrevistadas que relataram o uso do sulfato ferroso expressaram o enfretamento de dificuldades relacionadas à administração do medicamento e as reações adversas por ele provocadas; mas, expressaram também a insistência no uso do medicamento.

Eu acho que o problema é o gostinho, né? Percebo que é meio estranho, no início ele estranhou, depois tomou. Ele só rejeitava, mas como eu sabia que era para o bem dele, eu insisti. (E6)

Nas primeiras vezes ela não tomava o suco de jeito nenhum, e o remédio estava junto com o suco. Depois de insistir muito é que ela tomava. E as fezes dela ficou bem escura. (E2)

Ele tá até tomando. Assim, tá e não tá, né? Porque ele tá tendo reação e está vomitando, não para no estômago. De tudo quanto é jeito, já preparei na seringa, na colher, no copo, no meio do suco, no meio da fruta. (E1) 
É dificil, porque na maioria das vezes ele joga o remédio fora. E também eu notei que, depois que dei o remédio, a cor do cocô mudou para uma cor preta meio verde. (E4)

Das crianças que não tinham prescrição do sulfato ferroso, algumas entrevistadas relataram conhecer o medicamento e questionaram a não prescrição do mesmo para a criança durante os acompanhamentos de saúde.

Eu fiquei sabendo que quando os nenéns começam a crescer eles passam [médicos prescrevem], não é? Que é pra dar no suco. Minha mãe sempre cuidava dos filhos aqui da vizinha, e sempre passavam e eles tomavam. Então, eu falei assim: "Mãe, passou o sulfato ferroso para ele [criança]?". Aí ela falou que não. (E10)

Quando eu levei ela [criança], eu questionei se precisava dar. Aí o médico falou que não, que ela estava bem. Quando você tá com anemia, eu acho que o sulfato ferroso é o que ajuda, né? Aí a médica disse que não precisava, que o sulfato ferroso não era pra evitar, era pra tratar. (E7)

\section{DISCUSSÃo}

Os resultados do estudo sugerem uma superficialidade no conhecimento das mães/cuidadores sobre AF, sua definição e prevenção, além da fragilidade em reconhecer a necessidade do uso do sulfato ferroso como medida preventiva.

Expressar um conceito a respeito da AF é uma dificuldade encontrada por algumas mães, demonstrada pela ausência de definição ou pela ocorrência de relação da doença com os sinais clínicos dela, além de frequentemente utilizarem a expressão "sangue fraco" para se referirem à anemia ${ }^{8}$. Ao expressarem seu conceito sobre a doença, as participantes traziam suas definições permeadas por dúvidas, caracterizando uma afirmação seguida de uma pergunta. Ainda, alguns relatos desvelam a ideia de que a anemia possa evoluir para o desenvolvimento da leucemia, relação já encontrada em outro estudo ${ }^{8}$, fato que sugere a necessidade de mais esclarecimentos e informações aos cuidadores. O pouco conhecimento sobre a enfermidade foi identificado como um fator dificultador para a prevenção da $A F$, pois se torna difícil atuar na prevenção de uma doença a qual as mães/cuidadores pouco conhecem.

Relacionar a AF com lacunas na alimentação foi um aspecto recorrente nas entrevistas, com destaque para a importância dos alimentos na prevenção da doença. Desse modo, tal aspecto é identificado como facilitador para a prevenção da enfermidade. Porém, estudo que investigou a prática alimentar de crianças demonstrou que tanto os alimentos fontes naturais de ferro quanto aqueles fortificados com o mineral foram ingeridos em baixas quantidades por crianças em seu primeiro ano de vida ${ }^{16}$. Ofertar alimentos variados é uma recomendação para crianças até os dez anos de idade ${ }^{17}$; porém, devido a condições econômicas, sociais e culturais, a diversificação nesse oferecimento não é uma realidade presente em diversas famílias brasileiras.
A experiência de ter vivenciado ou conhecer alguém acometido pela anemia trouxe bastante apreensão entre algumas entrevistadas, pois a evolução da doença e a vulnerabilidade das crianças podem trazer consequências à saúde delas. Por este fato, se faz necessário conhecer as consequências da enfermidade, para que se reflita sobre a prevenção da doença e a promoção da saúde das crianças ${ }^{8}$.

Com relação ao uso do sulfato ferroso, apreendeu-se dos relatos que algumas mães reconhecem a importância do medicamento na prevenção da anemia e que as principais dificuldades são: rejeição por parte da criança em ingerir o medicamento, suspeita de que o gosto é desagradável e o escurecimento das fezes. Porém, notou-se que apesar das dificuldades enfrentadas, as entrevistadas entendem os benefícios do medicamento para a saúde das crianças e insistiram em seu uso. Os efeitos colaterais advindos do uso do sulfato ferroso, além do esquecimento são apontados como fatores principais para a continuação ou até mesmo sua interrupção $0^{8-10}$. Por isso, famílias e cuidadores necessitam de orientações quanto a continuidade no uso do sulfato ferroso, como também de esclarecimentos prévios, sobre possíveis efeitos colaterais, visando à prevenção da doença e o uso correto do medicamento. Apesar de alguns relatos revelarem importância do sulfato ferroso, as dificuldades enfrentadas em seu uso, aliadas aos efeitos colaterais, são identificadas como aspectos que dificultam a prevenção da enfermidade, a semelhança de outros estudos ${ }^{8-10}$, nos quais os efeitos colaterais foram determinantes para a interrupção do medicamento.

A falta de monitoramento e acompanhamento da suplementação, por parte dos profissionais de saúde das ESF, também é um fator que influencia diretamente na suplementação com sulfato ferroso ${ }^{18}$. No presente estudo, além da prescrição, não houve relatos sobre o acompanhamento de profissionais de saúde quanto à sua utilização.

Embora algumas entrevistadas tenham apontado o uso do sulfato ferroso como medida preventiva da doença, investigações demonstraram compreensão da relação do medicamento somente com o tratamento da $\mathrm{AF}^{9}$ e baixo percentual de cuidadores que reconhecem o sulfato ferroso como medida preventiva da enfermidade $^{8}$. Tais fatores podem contribuir tanto para a baixa adesão ao sulfato ferroso quanto para a efetividade do programa de suplementação.

Problemas de planejamento e gestão, motivação de mães e de profissionais de saúde, a falta de capacitação dos profissionais e de prescrição do medicamento são alguns dos motivos apontados como entraves para a efetividade do Programa Nacional de Suplementação de Ferro ${ }^{7,19}$. Os relatos mostraram que algumas crianças não receberam a prescrição do medicamento, o que sugere uma atuação profissional dissonante das recomendações das políticas públicas, em prol da pre- 
venção da AF. O baixo número de crianças do estudo com a prescrição do sulfato ferroso, bem como a falta de orientação sobre seu uso foram identificadas como fatores que dificultam a prevenção da anemia, apesar das recomendações das políticas públicas e de as orientações favorecerem a prevenção da enfermidade. Além disso, as capacitações para os profissionais da saúde devem considerar suas crenças e experiências para que as recomendações sejam incorporados nos atendimentos ${ }^{20}$.

Considerando as lacunas e fragilidades na implementação programático-assistencial do Programa Nacional de Suplementação de Ferro encontradas neste estudo e que a AF pode ter consequências irreversíveis na saúde e no desenvolvimento da criança, podemos afirmar que estamos diante de um quadro de vulnerabilidade no desenvolvimento infantil. Ainda, as insuficiências nas implementações programáticas dificultam o acesso e privam os usuários de seus diretos, potencializando chances de prejuízos para a saúde e impactando negativamente na qualidade de vida das crianças $^{21}$.

Pesquisa realizada no município em que foi desenvolvido este estudo identificou, em 2010, uma prevalência de $48 \%$ da anemia na faixa etária de seis a doze meses de vida ${ }^{22}$. Esse dado reforça a importância de instituir ações de vigilância em saúde da criança, cabendo aos enfermeiros condutas que envolvam promoção da saúde e prevenção de agravos, intervenções de caráter educativo e assistencial e detecção precoce de situações vulneráveis. Nesse sentido, o cuidado da criança no âmbito da atenção primária à saúde objetiva assegurar o seguimento e a vigilância das necessidades essenciais, do crescimento e desenvolvimento infantil, considerando valores maternos e familiares ${ }^{23}$. Por isso, o cuidado integral e longitudinal à saúde da criança é peça fundamental dentro das ações desenvolvidas na ESF.

\section{CONCLUSÕES}

O presente estudo identificou aspectos relativos ao conhecimento de mães/cuidadores sobre a AF e sua prevenção, denotando deficiência e superficialidade nestes saberes, além de lacunas no acesso à prescrição do sulfato ferroso e de informações sobre sua utilização. Os resultados apontam para fragilidades da atenção à saúde da criança no contexto estudado, remetendo à privação de direitos em saúde e predisposição à uma situação de vulnerabilidade em saúde, o que pode favorecer o surgimento da AF e suas complicações a curto e longo prazo.

A alimentação saudável foi apontada como um elemento facilitador na prevenção da AF. A fragilidade nos conhecimentos sobre a enfermidade e o uso do sulfato ferroso como estratégia preventiva, falhas na prescrição do sulfato ferroso, dificuldades para o uso do medicamento e pouco repertório de orientações são elementos que dificultam a prevenção da doença.
Os resultados podem direcionar futuras práticas educativas em saúde com foco na prevenção da $A F$, pois indicam que informações sobre a doença e o uso cotidiano do sulfato ferroso em crianças são temas que merecem atenção e devem ser destaque para o cuidado da sua saúde, tanto para as famílias quanto para os profissionais de saúde. Acresce-se a relevância no monitoramento da rotina de suplementação profilática, tanto na prescrição quanto na adesão e interrupção.

A enfermagem tem papel fundamental na promoção de práticas educativas em saúde, orientando e esclarecendo dúvidas, auxiliando em tomadas de decisões e problemas. Na rotina da ESF, as consultas de puericultura e as visitas domiciliares devem ser consideradas práticas com oportunidades significativas, com interação e compartilhamento de saberes, em que a enfermagem pode atuar visando a prevenção de agravos, promoção, tratamento e recuperação da saúde infantil.

O presente estudo apresenta limitações pelo reduzido número de participantes e sua realização em uma realidade contextualizada. A abordagem utilizada traz aspectos relevantes à compreensão qualitativa do fenômeno da prevenção da AF em crianças e incentiva uma reflexão sobre a garantia de direitos e de acesso às políticas públicas de saúde instituídas para a população infantil. Por fim, gera aspectos a serem investigados em novas pesquisas em diferentes cenários e contextos.

Agradecimento: À Coordenação de Aperfeiçoamento de Pessoal de Nível Superior (CAPES), pelo fomento concedido (bolsa mestrado). Estudo vinculado à projeto de pesquisa CNPq Processo 309085/2015-2.

\section{REFERÊNCIAS}

1. Silva PWKA, Costa CMF, Natal FJ, Malaquias BF. Anemia e deficiência de vitamina $A$ em crianças menores de cinco anos assistidas pela Estratégia Saúde da Família no Estado de Pernambuco, Brasil. Ciênc. saúde coletiva [Internet]. 2014 [acesso em: 17 fev 2017]; 19(4): 1209-1222. Disponível em: http://dx.doi. org/10.1590/1413-81232014194.00602013.

2. Ministério da Saúde (Br), Ministério da Educação (Br). NutriSUS - Estratégia de fortificação da alimentação infantil com micronutrientes (vitaminas e minerais) em pó : manual operacional. 2015. [Internet]. Brasília (DF): Ministério da Saúde; 2015 [acesso em: 20 abr. 2017]. Disponível em: http://189.28.128.100/dab/docs/ portaldab/publicacoes/manual ferro2013.pdf

3. Ministério da Saúde (Br). Secretária de Atenção à Saúde. Departamento de Atenção Básica. Programa Nacional de Suplementação de Ferro - Manual de Condutas Gerais. [Internet]. Brasília (DF): Ministério da Saúde; 2013, [acesso em: 21 maio 2017]. Disponível em: <http://dab.saude.gov.br/portaldab/biblioteca. php?conteudo=publicacoes/manual_ferro2013>.

4. Ministério da Saúde (Br). Pesquisa Nacional de Demografia e Saúde da Criança e da Mulher - PNDS 2006. Capítulo 13: Micronutrientes. 2009. [Internet]. Brasília (DF): Ministério da Saúde; 2009 [acesso em: 22 jan. 2017]. Disponível em: <http://bvsms.saude.gov. $\mathrm{br} / \mathrm{bvs} /$ publicacoes/pnds_crianca_mulher.pdf $>$

5. Bortolini GA, Vitolo MR. Impacto de orientação dietética sistemática no primeiro ano de vida nas prevalências de anemia e deficiência de ferro aos 12-16 meses. J. Pediatr. (Rio J.) [Internet]. 2012 [acesso em: 22 jan. 2017]; 88(1): 33-39. Disponível em: 
http://dx.doi.org/0.2223/JPED.2156.

6. Ministério da Saúde (Br). Secretaria de Atenção à Saúde. Departamento de Atenção Básica. Manual operacional do Programa Nacional de Suplementação de Ferro. 2005. [Internet] Brasília (DF): Ministério da Saúde; 2005 [acesso em: 03 abr. 2017]. Disponível em http://189.28.128.100/dab/docs/portaldab/publicacoes/ manual_ferro.pdf.

7. Stulbach TE, Name JJ, Daboin BEG, Szarfarc SC. Efficacy of the national program of iron supplementation in the anaemia control in infants assisted by child education centers. Journal of Human Growth and Development. [Internet] 2014 [acesso em: 22 jan. 2017]; 24(3):282-288. Disponível em: http://dx.doi.org/10.7322/ jhgd.88960.

8. Azeredo CM, Cotta RMM, Silva LS, Franceschini SCC, Sant'Ana LFR, Lamounier JA. A problemática da adesão na prevenção da anemia ferropriva e suplementação com sais de ferro no município de Viçosa (MG). Ciênc. saúde coletiva [Internet]. 2013 [acesso em: 17 set. 2017]; 18(3): 827-836. Disponível em: http://dx.doi. org/10.1590/S1413-81232013000300028.

9. Machado JS, Nunes JS, Nunes GBL. Saberes e práticas maternas relacionadas à suplementação do ferro em crianças de 6 à 18 meses. Rev. baiana enferm. [Internet] 2014 [acesso em: 12 mar. 2018]; 28(1): 13-22. Disponível em: http://dx.doi.org/10.18471/ rbe.v28i1.8315.

10. Gontijo TL, Oliveira VC, Lima KCB, Lima PKM. Prática Profilática da Anemia Ferropriva em Crianças na Estratégia Saúde da Família. Revista de Enfermagem do Centro-Oeste Mineiro [Internet] 2017 [acesso em: 28 fev. 2018]; 7:e1204. Disponível em: http://dx.doi. org/10.19175/recom.v7i0.1204

11. World Health Organization (WHO). Iron Deficiency Anaemia: Assessment, Prevention, and Control - A guide for programme managers. 2001 [Internet]. Geneva: United Nations Children's Fund/United Nations University/World Health Organization; 2001 [acesso em: 11 mar 2017]. Disponível em: http://apps.who.int/ iris/ bitstream/10665/66914/1/WHO_NHD_01.3.pdf.

12. Sociedade Brasileira de Pediatria (SBP). Departamento de Pediatria Ambulatorial. Manual Prático de Atendimento em Consultório e Ambulatório de Pediatria. 2006 [Internet]. São Paulo: SBP 2006 [acesso em: 18 nov. 2016]. Disponível em: http://www.sbp. com.br/fileadmin/user_upload/2015/02/ManPraticaAtend.pdf. 13. Ministério da Saúde (Br). Secretaria de Atenção à Saúde. Departamento de Atenção Básica. Dez passos para uma alimentação saudável: guia alimentar para crianças menores de dois anos: um guia para o profissional da saúde na atençãobásica. 2010 [Internet]. Brasília (DF): Ministério da Saúde; 2010 [acesso em: 18 nov. 2016]. Disponível em: http://www.redeblh.fiocruz.br/ media/10palimsa_guia13.pdf.

14. Ministério da Saúde ( $\mathrm{Br}$ ). Secretaria de Atenção à Saúde. Departamento de Atenção Básica. Saúde da criança: aleitamento materno e alimentação complementar. Capítulo 3: Duração da amamentação. 2015 [Internet] Brasília (DF): Ministério da Saúde;
2015 [acesso em 18 nov. 2016]. Disponível em: http://bvsms. saude.gov.br/bvs/publicacoes/saude_crianca_aleitamento_materno cab23.pdf.

15. Braun V, Clarke V. Using thematic analysis in psychology. Qualitative Research in Psychology [Internet] 2006 [acesso em: 21 ago. 2016] ; 3(2): 77-101. Disponível em: http://dx.doi. org/10.1191/1478088706qp063oa.

16. Alleo LG, Souza SB, Szarfarc SC. Feeding practices in the first year of life. Journal of Human Growth and Development [Internet] 2014 [acesso em 27 nov. 2017]; 24(2): 195-200. Disponível em: http://pepsic.bvsalud.org/scielo.php?script=sci arttext\&pid=S0104-12822014000200012\&lng=pt\&tlng=pt.

17. Ministério da Saúde $(\mathrm{Br})$. Secretaria de Atenção à Saúde. Departamento de Ações Programáticas Estratégicas. Caderneta de Saúde da Criança. 2017 [Internet] Brasília (DF): Ministério da Saúde; 2017 [acesso em 15 mar. 2018]. Disponível em: http:// bvsms.saude.gov.br/bvs/publicacoes/caderneta_saude_crianca_menina_11ed.pdf.

18. Azeredo CM, Cotta RMM, Silva LS, Franceschini SCC, Sant'Ana LFR, Ribeiro RCL. Implantação e impacto do Programa Nacional de Suplementação de Ferro no município de Viçosa - MG. Ciênc. saúde coletiva [Internet]. 2011 [acesso em: 17 set. 2017]; 16(10):4011-4022. Disponível em: http://dx.doi. org/10.1590/S1413-81232011001100006.

19. Cembranel F, Corso ACT, González-Chica DA. Cobertura e adequação da suplementação com sulfato ferroso na prevenção de anemia em crianças atendidas em centros de saúde de Florianópolis, Santa Catarina. Rev. paul. pediatr. [Internet]. 2013 [acesso em 07 dez. 2017]; 31(3): 315-323. Disponível em: http://dx.doi. org/10.1590/S0103-05822013000300007.

20. Palombo CNT, Fujimori E, Toriyama ATM, Duarte LS, Borges ALV. Difficulties in nutritional counseling and child growth follow-up: from a professional perspective. Rev. bras. enferm. [Internet] 2017 [acesso em 29 mai. 2018]; 70( 5 ): 949-957. Disponível em: http:// dx.doi.org/10.1590/0034-7167-2016-0527.

21. Silva DI, Veríssimo MLOR, Mazza VZ. Vulnerability in the child development: influence of public policies and health programs. Journal of Human Growth and Development [Internet]. 2015 [acesso em 28 mai. 2018]; 25(1): 11-18. Disponível em: http:// dx.doi.org/10.7322/jhgd.96760.

22. Reis MCG, Nakano AMS, Silva IA, Gomes FA, Pereira MJB. Prevalência de anemia em crianças de 3 a 12 meses de vida em um serviço de saúde de Ribeirão Preto, SP, Brasil. Rev. latinoam. enferm. [Internet]. 2010 [acesso em: 01 mar 2018] 18(4): 792-799. Disponível em: http://dx.doi.org/10.1590/S010411692010000400019.

23. Mello DF, Henrique NCP, Pancieri L, Veríssimo MLÓR, Tonete VLP, Malone M. Child safety from the perspective of essential needs. Rev. latinoam. enferm. [Internet]. 2014 [acesso em 28 mai. 2018]; 22( 4 ): 604-610. Disponível em: http://dx.doi. org/10.1590/0104-1169.3651.2458. 